\title{
MASSIVE-SCALE TREE MODELLING FROM TLS DATA
}

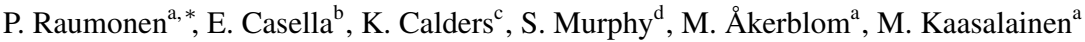 \\ a Tampere University of Technology, Tampere, Finland- (pasi.raumonen, mikko.kaasalainen, markku.akerblom)@ tut.fi \\ ${ }^{\mathrm{b}}$ Forest Research, Farnham, UK- eric.casella@forestry.gsi.gov.uk \\ ${ }^{c}$ Wageningen University, Wageningen, Netherlands- kim.calders@wur.nl \\ ${ }^{\mathrm{d}}$ Melbourne School of Land and Environment, University of Melbourne, Australia- murphys@ unimelb.edu.au
}

KEY WORDS: Quantitative structure models, automatic tree extraction, biomass, forest plot, ground truth, oak, eucalyptus, LiDAR

\begin{abstract}
:
This paper presents a method for reconstructing automatically the quantitative structure model of every tree in a forest plot from terrestrial laser scanner data. A new feature is the automatic extraction of individual trees from the point cloud. The method is tested with a 30-m diameter English oak plot and a 80-m diameter Australian eucalyptus plot. For the oak plot the total biomass was overestimated by about $17 \%$, when compared to allometry $(\mathrm{N}=15)$, and the modelling time was about 100 min with a laptop. For the eucalyptus plot the total biomass was overestimated by about $8.5 \%$, when compared to a destructive reference $(\mathrm{N}=27)$, and the modelling time was about $160 \mathrm{~min}$. The method provides accurate and fast tree modelling abilities for, e.g., biomass estimation and ground truth data for airborne measurements at a massive ground scale.
\end{abstract}

\section{INTRODUCTION}

The measurement of above-ground forest biomass is important for ecology, carbon storage estimation, forest research, etc. Currently airborne and satellite-based remote sensing is needed to estimate the biomass of large forest areas. These measurements require calibration based on good quality reference or ground truth data from test sites. Furthermore, it should be possible to measure the reference data quickly, cheaply, and nondestructively. The traditional way is to use allometry, but this can be problematic as errors can be in some cases over 30\% (Calders et al. 2015).

With terrestrial laser scanning (TLS) and computational methods it is now possible to automatically reconstruct accurate and precise 3D models of the tree structure of the scanned trees in minutes (Raumonen et al. 2013, Calders et al. 2015). We call these models quantitative structure models or QSMs. They represent the trees as hierarchical collections of cylinders or other building blocks which provide the volume and diameter of branch segments needed to estimate the biomass. From QSMs it is thus possible to accurately and non-destructively estimate the volume, branching structure and branch size distribution and also detect growth and changes (Kaasalainen et al. 2014). Notice that the building blocks approach makes the models discontinuous, but this does not affect the modelled structural parameters. Overall, the circular cylinder is the best choice for a block type ( $\AA$ kerblom et al. 2014).

Our method and most other automatic reconstruction methods so far have been working only for scans with single trees, i.e., a manual extraction of the trees from the scans was required. In this paper we present automatic reconstruction for plot level. In other words, we use the "raw data" of the scans, which contain all the trees in the plot and measurements from ground and understory. The scans are only registered into a common coordinate system and then all the individual trees are automatically extracted and the QSMs are reconstructed. Fig. 1 shows an example of tree extraction and QSM reconstruction for a plot with a $15-\mathrm{m}$ radius (modelling time about $100 \mathrm{~min}$ with a laptop).

\footnotetext{
${ }^{*}$ Corresponding author
}

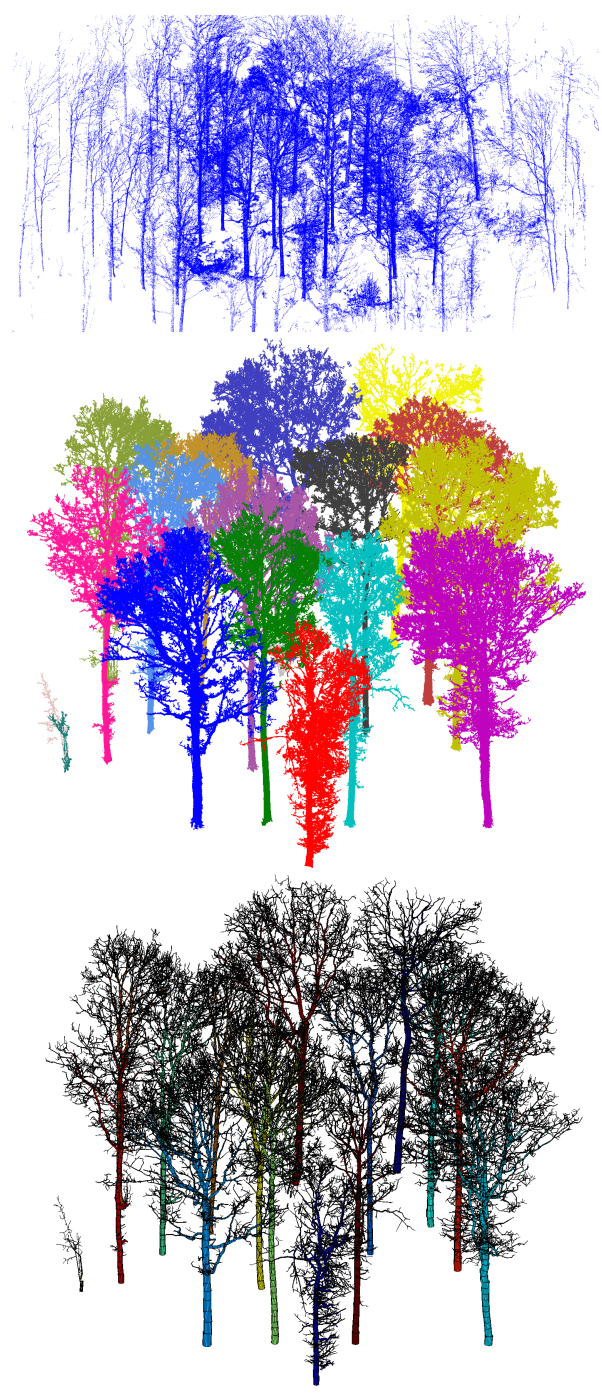

Figure 1. Filtered (top) and tree-segmented (middle) point cloud and the reconstructed QSMs (bottom) of an English oak plot. 
Plot-level tree modelling is thus very fast and also makes the tree extraction faster and more reliable. Extracting individual trees manually from large point clouds is time consuming (a couple of hours for one oak tree), and if the branches of neighbouring trees overlap, the manual separation is not easy or reliable. There are of course challenges also in automatic tree extraction, and it is probably impossible to make an algorithm that could always extract trees without any errors, especially for dense forests. We demonstrate and test our method with an English oak leaf-off plot and an Australian leaf-on eucalyptus plot.

Very large point clouds may require algorithms optimising memory usage and computation times. For example, parallel computing can be used to simultaneously reconstruct the QSMs for each tree. Our demonstration uses $15-\mathrm{m}$ and $40-\mathrm{m}$ radius plots, but the method can be easily applied to much larger areas while the memory and computation time increase linearly with the plot area. Thus it is possible to scan and automatically model, e.g., multi-hectare-size plots.

There are a number of other methods for reconstructing single trees from TLS-data, e.g. (Dassot et al 2012, Hackenberg et al. 2014), but these require (semi-)manual tree extraction. There are automatic methods for some inventory data, such as dbh, volume and height, from TLS data, e.g. (Hopkins et al. 2004, Maas et al. 2008). Eysn et al. (2013) presented a fast but manual method for separating the stems and biggest branches from plot-level data.

The paper is organised as follows. In section 2 we introduce the data, present the details of the extraction and reconstruction method and a sensitivity analysis. In Section 3 we present the results, and sections 4 and 5 contain discussion and conclusions.

\section{MATERIALS AND METHODS}

In this section we first present the data used for the examples, then we give an outline of the reconstruction method, present the details of the tree extraction and reconstruction methods, and finally go over some sensitivity analysis.

\subsection{Measurements}

We have measurements from two contrasting forest plots collected with different scanners and setups. The first plot is an 80 -year-old oak plantation in South-Eastern England (51.1533 $\mathrm{N} ; 0.8512^{\circ} \mathrm{W} ; 82 \mathrm{~m}$ asl). The plantation covers a total ground area of about 85 ha, managed as a commercial forest of mixed species dominated at $75 \%$ by Quercus robur L. The stocking density was 405 trees per ha in 2011, with a mean height of $23+/-2$ $\mathrm{m}$. The understory was dominated by hazel (Corylus avellana L.) and hawthorn (Crataegus monogyna Jacq.) bushes. A $30 \mathrm{x}$ $30 \mathrm{~m}$ experimental area was selected and the understory removed before data acquisition. We have a reference for dry mass ( $D M$, in kilograms) given by an empirical relation computed from the diameter at breast height $(D B H$, in meters):

$$
D M=14720 D B H^{2.8682} .
$$

The wood density used for converting the modelled volume to dry mass is $507+/-40 \mathrm{~kg} / \mathrm{m}^{3}$. Five scan locations was used for this study, one in the centre and the others near the boundary of the plot. They were recorded in April 2012 (leaf-off, no precipitation, wind speed averaged over the acquisition period was $0.7+/-0.5$ $\mathrm{m} / \mathrm{s})$.

The other plot is part of native Eucalyptus open forest (dry sclerophyll Box-Ironbark forest) in Victoria, Australia $\left(36^{\circ} 45^{\prime} 36.49\right.$ " $S$; $145^{\circ} 0^{\prime} 59.58^{\prime \prime} E$ ). The plot, called RUSH06, has a 40 -m radius and it was established in Rushworth forest and partially harvested in May 2012 to acquire accurate estimates of biomass. The main tree species in the plot were Eucalyptus leucoxylon, E. microcarpa and E. tricarpa. Five scan locations, with one in the centre and four $40 \mathrm{~m}$ from the centre, were used (Calders et al 2015).

The oak plot was scanned with the field portable $(15 \mathrm{~kg})$, phaseshift range finder, single return, HDS-6100 (Leica Geosystems Ltd., Heerbrugg) with a rotating mirror system that can cover a $310^{\circ}$ in zenith by $360^{\circ}$ in azimuth field of view. The beam wavelength is within the red band $(650-690 \mathrm{~nm})$ with a $0.003 \mathrm{~m}$ spot size at exit and a divergence angle of $0.013^{\circ}$. The angular sampling resolution was $0.036^{\circ}$ in both directions. The maximum detection range is about $80 \mathrm{~m}$. The scanner was mounted on a tripod with the laser source located at about $1.3 \mathrm{~m}$ above the ground. For each location, one full scan $\left(310^{\circ} \times 360^{\circ}\right)$ was recorded. The final co-registered point cloud has about 124 million points.

The eucalyptus plot was scanned with a RIEGL VZ-400 (RIEGL Laser Measurement Systems $\mathrm{GmbH}$ ). The beam divergence is $0.02^{\circ}$ and the instrument operates in the infrared band $(1550 \mathrm{~nm})$ with a detection range up to $350 \mathrm{~m}$. The angular sampling resolution was $0.06^{\circ}$ in both directions. The scanner allows fast data acquisition and records multiple return LiDAR data (up to four returns per emitted pulse). The advantage of multiple returns over single returns is the improved sampling at greater canopy heights (Calders et al. 2014). Because RIEGL VZ-400 has a zenith range of 30 to 130 degrees, an additional scan was acquired at each scan location with the instrument tilted at 90 degrees to sample a full hemisphere. The final co-registered point cloud has about 71 million points.

For both plots, reflecting targets (six $15 \mathrm{~cm}$ black \& white tilt \& turn targets for the oak plot) were distributed throughout the plot area and were used to co-register the five scan locations using the Cyclone (Leica Geosystems, version 9) and the RiSCAN PRO (provided by RIEGL). The error in the co-registration of point clouds was $0.125+/-0.075 \mathrm{~cm}$ for the Oak plot (individual scans ranging from $0-3 \mathrm{~mm}$ ), and $1.29 \mathrm{~cm}$ (ranging from $0.62 \mathrm{~cm}$ to $2.26 \mathrm{~cm}$ ) for the eucalyptus plot.

\subsection{Outline of the Method}

The method is based on morphological rules, a cover-set approach, and geometric primitives. By morphological rules we mean that the reconstruction steps follow the tree topology and the major shape characteristics. The cover-set approach means a technical aspect of how point clouds are filtered and segmented, first into trees and then into branches, by concentrating on the smallest meaningful parts or units (surface patches) on the tree surface. The surfaces and structures of the trees are reconstructed with suitable geometric primitives; in this paper with cylinders.

The first major but optional step in the method is filtering. We filter out noise, remove some ground and understory points, include only the points within a given plot size, and make regions of very high point density sparser (to save memory).

The second major step is the extraction of individual trees from the filtered point cloud. It is based on the segmentation of the point cloud into stems and branches using large surface patches of a fixed size. The stem bases and approximate stems are located heuristically, based on the assumption that stems are vertical. The likely ground and understory patches are defined similarly. The other patches are classified as either tree or other (ground, understory, etc.) based on their connectedness and distance from classified patches. Patches classified as trees are next segmented 
into individual trees following botanical rules: first determine the stems one-by-one, then the 1st-order branches one-by-one, then the 2nd-order branches, etc. In cases where the trees are separated from each other by large enough distances between their closest branches, the segmentation process extracts the individual trees reliably. When there are some contacts between the branches from different trees (or the gaps between the trees are small), we can still expect a reasonably good separation of trees. At this point further heuristic correction steps can be included.

The third and final major step is the reconstruction of QSMs for each tree individually. The QSM reconstruction has been validated earlier (Calders et al. 2015). Again the point cloud is covered with patches, this time with smaller and variable size that decreases as the branches get smaller. The variable size is based on the information from the first segmentation used for tree extraction. The patches are again segmented into stems and branches as earlier, and finally each segment is partitioned into smaller regions for cylinder fitting.

We have made a short video demonstrating the method (Åkerblom 2014).

\subsection{Technical tools}

Before going into the details of the major steps in the method, we discuss briefly some technical but necessary tools we need.

2.3.1 Cubical partition To handle a large number of points effectively without requiring huge amount of memory and to avoid pointless computations, it is useful to partition the point clouds into smaller cubical regions. The coordinates $Q$ of the cubical region where each point belongs are computed from 1) the coordinates $P$ of the points; 2 ) the "lowest corner" $C_{L}$ of the cubical partition space (usually the minima of the $\mathrm{x}, \mathrm{y}, \mathrm{z}$-coordinates); and 3 ) the edge length $e$ of the cubes: $Q=$ floor $\left[\left(P-C_{L}\right) / e\right]+1$. Here floor denotes rounding to the nearest smaller integer, and the three components $\left[Q_{X}, Q_{Y}, Q_{Z}\right]$ of $Q$ are between 1 and the number of cubes $\left(N_{X}, N_{Y}, N_{Z}\right)$ in the sides of this cubical space. One way to quickly determine the points in the same cubes is to use the lexicographical coordinate $L$ of $Q: L=$ $Q_{X}+\left(Q_{Y}-1\right) N_{X}+\left(Q_{Z}-1\right) N_{X} N_{Y}$. Points are sorted into increasing $L$-values and the consecutive points with the same $L$ values are selected to form each cube.

For a fast way of finding neighbouring cubes, the $e$-cubes should be recorded also as a 3D-cell array, where the coordinates $Q$ give also the cell coordinates. However, with small $e$-values compared to the size of the space, the number of cubes can be so large that there is not enough memory to store all the cells. Fortunately, because the points are from the surfaces of trees, the whole space is often quite sparsely filled. Thus it is possible to partition the space first into much larger cubes with an edge length $E$, e.g. 1-10 $\mathrm{m}$, in which case the number of $E$-cubes is only a small fraction compared to $e$-cubes and still most of the $E$-cubes will be empty. Then we select only the nonempty $E$-cubes and expand them to include one layer of neighbouring $e$-cubes from the neighbouring $E$-cubes. The point cloud is thus partitioned into an array of nonempty $E$-cubes, which are further partitioned into $3 \mathrm{D}$-cell arrays of empty and nonempty $e$-cubes.

2.3.2 Cover Sets The cover sets of a point cloud sampling the surfaces of trees and other objects are small subsets corresponding to surface patches on the surfaces of the objects. The idea is to partition the surfaces into small sets that 1) are in some sense the smallest meaningful subsets corresponding to the details of the branching structure, and 2) conform to the local shape of the surface, i.e., correspond to connected surface patches. This is in contrast to voxel approaches, where the boundaries of the voxels are completely arbitrary with respect to the shape and the exact location of the details of the surface.

The process has two basic steps and the sets are randomly but about evenly covered along the surface of the tree with some restrictions. First we generate $r$-balls such that 1 ) the centres of the balls have at least a distance $d$ between them such that $d \leq r$ holds, 2) each point is at most the distance $d$ away from its closest centre, and 3) an acceptable ball has at least $n$ points. These balls can now overlap, which is exploited later. Second, each ball defines a cover set as those points of the ball that are closest to its centre. The cover sets thus do not overlap but form a partition of the points. Finally we define neighbour relations for the cover sets: two cover sets are neighbours if their $r$-balls intersect. This neighbour relation can be used as a convenient tool for expanding or moving along the surface or for determining connected components (Raumonen et al. 2013).

The generation can be realised using a cubical partition of points into $r$-cubes. To determine the $r$-ball for the point $p$, we take the $r$-cube of $p$ and its 26 neighbouring cubes to restrict the search to nearby points. More details can be found in Raumonen et al. 2013. To save memory with very large point clouds, it is possible to carry out a cover generation without saving the partition: when a large $E$-cube containing its $r$-cubes is defined, use it directly for the cover generation of that cubical region and then proceed to define the next $E$-cube and its cover generation.

\subsection{Filtering}

The first major step in the method is the filtering of the points that are not detections of a tree surface. Another aim here is to reduce the size of the point cloud so that the memory and computation time requirements can be reduced. First we remove points from ground and small understory as well as points that are too far away from the plot centre. Second, especially for high resolution scans the point density near the scanner may be unnecessarily high, and to reduce the number of points, we randomly remove some of these points. Finally, we try to remove noise, i.e., points that are not clear samples of any surface but are generated by the scanning process itself, e.g., by an error on range estimates due to the footprint of the laser hitting a limb.

First the point cloud is restricted by removing all the points whose horizontal distance from the plot's centre is $20 \mathrm{~m}$ over the plot's radius. Next the points are partitioned into horizontally defined rectangular regions and the bottom (ground and understory) is removed: The side length of these rectangles is set to, e.g., 0.5 $\mathrm{m}$ and the minimum and maximum z-values $Z_{L}$ and $Z_{H}$ for each rectangle are determined. If $Z_{H}>Z_{L}+0.5 \mathrm{~m}$ holds, we remove the points for which $Z<Z_{L}+0.25$ m holds.

Next we use the cubical partition of the already filtered point cloud to modify regions with too small or too high point densities: remove the points in cubes that have fewer points than some given threshold, and thin out cubes with more points than some threshold by randomly selecting a smaller sample. Here the cubes are considered separately without considering their neighbours. For the eucalyptus plot we used $10 \mathrm{~cm}$ cubes with 7 points as the minimum threshold and 150 points as the maximum one. The oak plot has a higher resolution: we used $10 \mathrm{~cm}$ cubes with 10 points as the minimum and 150 points for the maximum.

Finally we also removed 1) low density regions and 2) small separate clusters using the cover sets (Raumonen et al 2013). The point cloud is partitioned into regions with, e.g., $10 \mathrm{~m}$ times 10 $\mathrm{m}$ horizontal area, and each region is individually covered with 
$r$-balls with $r$ also as the minimum distance between the centres and the maximum distance from any point to its closest centre. For both plots we used $r=6 \mathrm{~cm}$. Then all the points that belong to a ball with more points than some given threshold (5 was used for both plots) are kept and others removed. Notice that a point may belong to multiple overlapping balls and it is assigned to the ball containing the most points. After this a new cover of the filtered point cloud using new set of parameters $(r, d)$ is generated. The connected components of this cover are determined and all components with less sets than some given threshold are removed. For both plots we used $r=6 \mathrm{~cm}, d=5 \mathrm{~cm}$ and the threshold was 30 sets per components.

The filtered oak plot has about 35 million points and the process lasted about $10 \mathrm{~min}$, while the filtered eucalyptus plot has about 33 million points and the process lasted about 16 min.

\subsection{Extraction of individual trees}

The next major step in the method is the extraction of individual trees from the point cloud. The idea is not to recover every little detail of the trees but only extract them quickly and reliably. Thus the size of the cover sets is not so important and large-size sets are used. The cover parameters at this stage are denoted as $d_{1}, r_{1}$, $n_{1}$. For the oak plot we used the values $d_{1}=8 \mathrm{~cm}, r_{1}=9 \mathrm{~cm}$, $n_{1}=3$, and for the eucalyptus plot, which has a lower resolution, we used the values $d_{1}=10 \mathrm{~cm}, r_{1}=12 \mathrm{~cm}, n_{1}=3$.

2.5.1 Locating stems and their bases The extraction starts by locating the stems. First the ground level is defined by partitioning the cover sets into $0.25 \mathrm{~m}$ horizontal squares and defining the ground level for each square as the lowest z-coordinate in its $5 \times 5$ neighbourhood. The "height" for each square is defined similarly. Next the surface normals of the cover sets up to $3 \mathrm{~m}$ above the ground are determined as the smallest principal component. The sets whose angle between the normal and the $\mathrm{z}$ direction is over $\alpha$ are selected as the most likely sets from stems (see the top of Fig. 2). We set $\alpha$ to 70 degrees. From these sets, with approximately horizontal normals, connected components are determined and components with over $\kappa$ sets are selected as potential stems (see the middle of Fig. 2). We set

$$
\kappa=\operatorname{ceil}\left[\frac{0.2}{\left(1.4 d_{1}\right)^{2}}\right]
$$

where ceil is the rounding to the nearest bigger integer. These components are now very probably part of the stems, but multiple components can be from the same stem. The components whose centres are horizontally closer than $\delta$ to each other are combined into one. We set $\delta$ to $20 \mathrm{~cm}$. Finally the bottom $25 \mathrm{~cm}$ of each component is removed as the potential layer containing ground, understory, and buttresses. Those components that still have at least $\kappa$ sets and whose vertical height is over $\lambda=1 \mathrm{~m}$ are selected as the final locations of the stems (see the bottom of Fig. 2).

Next the bases of the stems are determined. A cylinder giving the approximate stem diameter is fitted for each stem component. The component is then restricted to its bottom $70 \mathrm{~cm}$ and is expanded downwards with neighbours, and only sets with normals over $\alpha$ are accepted. After each expansion the cylinder is fitted again. The expansion is stopped when 1) the diameters of the cylinders are almost equal, or 2) the diameters are very different, or 3 ) the bottom is under $10 \mathrm{~cm}$ from the ground level. Then the bottom $50 \mathrm{~cm}$ of the expanded component is the base of the stem (see the top of Fig. 3). The above process has four parameters; $\alpha, \kappa, \delta$, and $\lambda$, but it is insensitive to quite large changes in their values as shown in the section 2.7 .

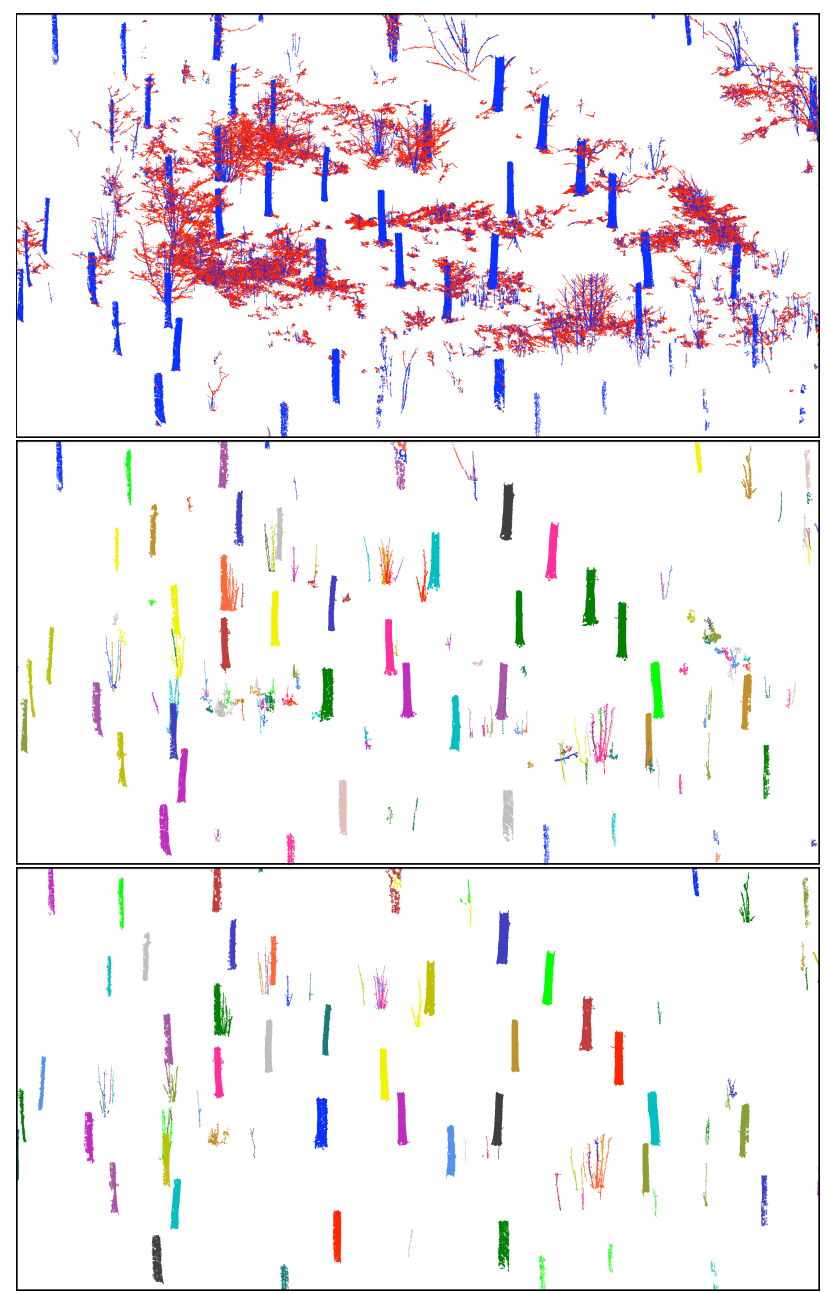

Figure 2. Locating stems. Top: "Stem-like" cover sets whose normals are nearly horizontal (blue) and the other cover sets (red) of the bottom $3 \mathrm{~m}$. Middle: The initial components of "stem-like" sets. Bottom: The final stem components which define the stems.

Because the extraction is based on segmentation, it is essential to segment also trees whose stem is outside but near the plot's boundary. Therefore all stems whose distance from the plot's centre is less than $R_{\text {plot }}+10 \mathrm{~m}$ are segmented (the red stems in Fig. 3) and all sets further than $R_{p l o t}+15 \mathrm{~m}$ are classified as other (the green stems in Fig. 3).

2.5.2 Classifying sets The next step is to classify every cover set either as tree or other. We begin by expanding the stem components upwards to determine the stems approximately (see the bottom of Fig. 3). We want approximate stems for robust tree extraction and the determination of initial ground and understory sets. To prevent an expansion too much downwards along branches or into understory or ground, the expansion is restricted to neighbours that are, e.g., under $50 \mathrm{~cm}$ from the current highest set. Due to occlusion this expansion may stop before the top of the tree. In such a case the nearest sets above the current top of the expansion are connected to the top and the expansion is continued until the top of the tree is reached.

The ground and understory sets are next defined as the non-stem sets in the 1-m layer above the ground level. These sets are then expanded as much as possible with the neighbours. At this point every set is classified as tree (stem) or other (ground and understory), or is not classified (see Fig. 4).

The unclassified sets are next classified by connecting them either 


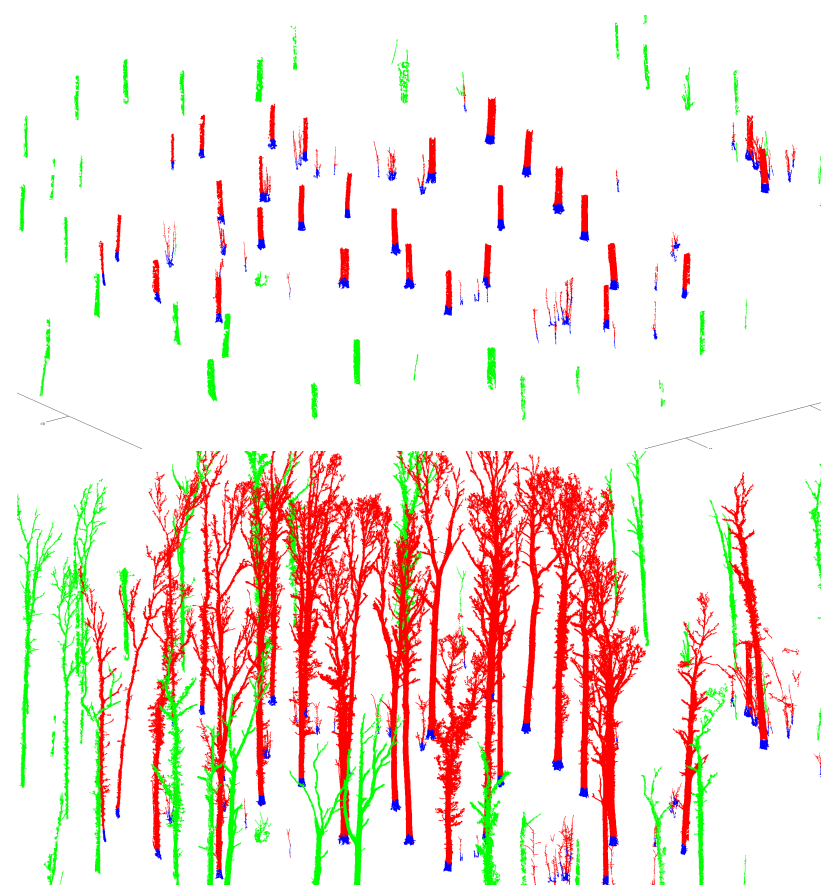

Figure 3. Approximate stems and their bases. Top: The stem components of Fig. 2 are expanded downwards and their bottom part (blue) is selected as the base. Green components are too far away from the plot's centre. Bottom: The stem components are expanded upwards to define the stems approximately.

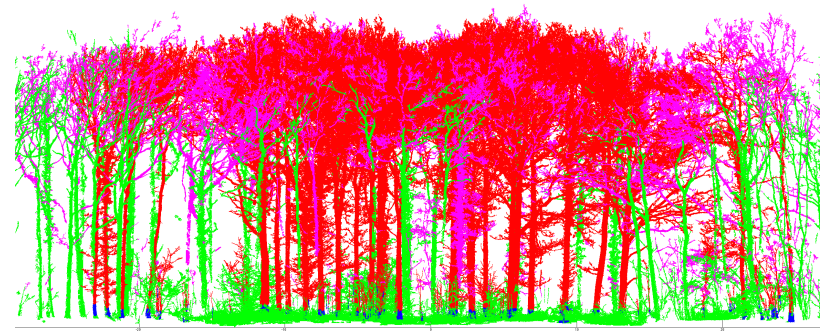

Figure 4. Classification of sets. Tree (red) and other (green) expanded as much as possible. Purple points are the non-classified sets which will be classified either as tree or other.

to tree or other sets. First we expand the tree sets into unclassified sets as much as possible. If there still are unclassified sets, then we connect them to either class. We determine the connected components of the unclassified sets and using the cubical partition, with initially about $20 \mathrm{~cm}$ cubes, we check if each component has in its neighbouring cubes tree or other sets. A component is connected to the closest tree or other set. In this process we first connect the nearest components, which then expand the tree and other sets step-by-step. If there are gaps so large that some components cannot be found in the neighbouring cubes of expanding tree or other sets, then we increase the size of the cubes as long as needed.

2.5.3 Segmentation of trees For the extraction of individual trees from the tree sets we use the segmentation method presented in Raumonen et al. (2013). We start from one of the bases of the stems and expand it step-by-step with neighbours. If the expanding "front" becomes disconnected, then there may be a bifurcation, in which case a new branch basis is defined and further expansion into that branch is prevented. The stem is defined by expanding it as much as possible, after which the same is done for the other stems. The process continues case-by-case from the bases of the first-order branches, and so on from the second-order

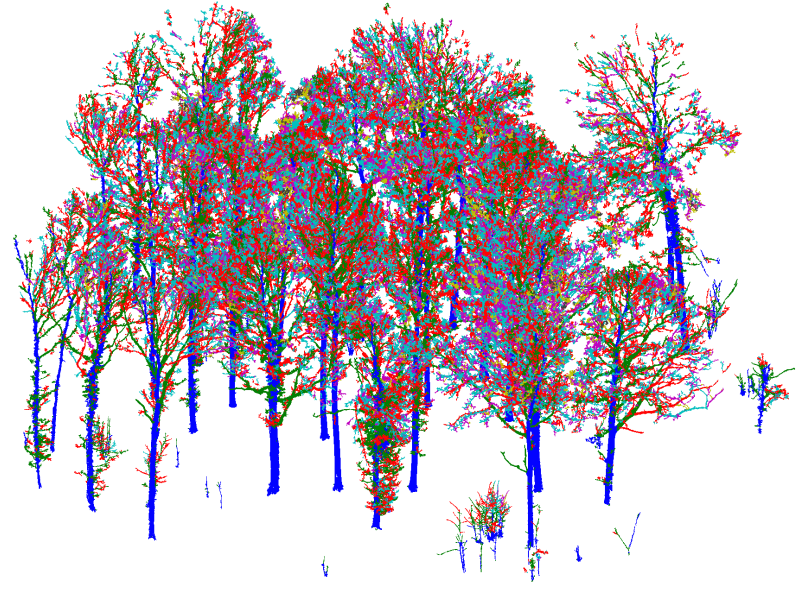

Figure 5. Segmentation of the tree sets. Stems are in blue, 1storder branches in green, 2nd-order branches in red, etc.

branches, etc., until every tree is segmented.

This initial segmentation may have problems: segments stop too early, or take wrong turns at bifurcations, or small segments could be part of bigger segments. We try to make some modifications to make the segmentation more correct, i.e., such that each segment corresponds to a real stem or branch without child branches. First we try to make the segments as long as possible, to correct the early stops or wrong turns. For each segment we select all the segments that can be traced back to it with the parent relation of the segments. Then we calculate the distances from the tips of these segments to the base of the current segment. If the furthest tip of these segments is farther than the tip of the current segment is from its base, we modify the segments and their parent-child relations so that the current segment continues without bifurcations to the furthest tip. The modification conforms to the branching order, i.e. first the stem, then the first-order branches, etc. For stems and first-order branches we use restrictions on the length vs. distance ratios making the segments nearly straight. Fig. 5 shows an example of the final segmentation of the point cloud, and the middle of Fig. 1 shows the extracted individual trees inside the plot.

There can be some problems in the resulting tree extraction. Due to occlusion, some tree parts may be quite far away from any other tree parts, and in our process this part is then connected to the closest tree part, which may not be the right one. In these situations, based on the distances from the stems and other information, one can try to estimate the likelihood that the part is connected to the right tree. We have not applied such methods in practice. Furthermore, two segments from different trees may touch each other, and because of their generation process, there is no guarantee that this connecting place is accurate. One frequent reason for this is that there is an erroneous connection made between tree parts and one tree reaches the other over a big gap. If this is the case, then we can expand through neighbours from the enjoining location into both trees and see if there are big gaps between neighbouring cover sets (see Fig. 6).

Finally we have the trees extracted and we can proceed to the reconstruction of the individual QSMs for the trees located inside the plot. However, in the tree extraction step the idea was not to model every detail and so the size of the cover sets was large everywhere. Smaller cover sets are needed to model smaller details. The segmentations used for the extraction give us approximations on the size of the branches and thus we can generate cover sets whose size is better suited for the local details. We calculate the local size for every tree point as follows: each segment is layered 


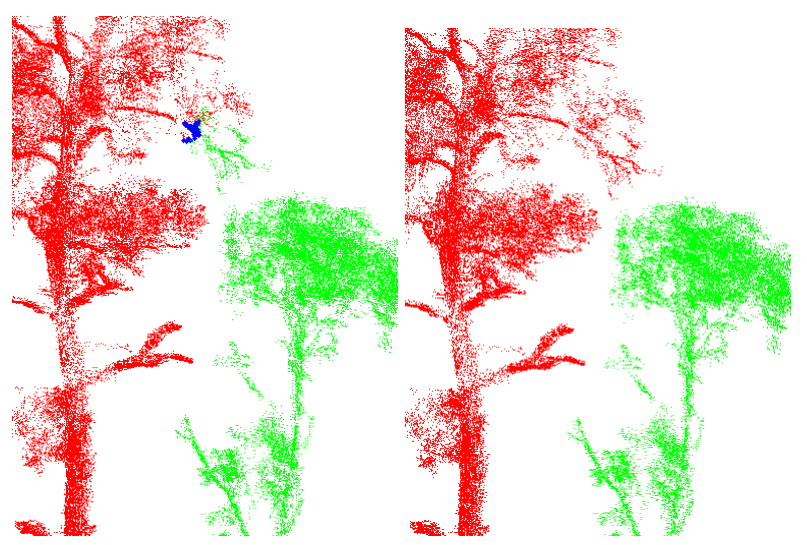

Figure 6. Tree extraction correction. Left: Blue denotes the meeting of two trees (red and green). Right: The expansion from the meeting place into the green tree is stopped earlier than the expansion into red tree because of a big gap and thus this expanded part is added to the red tree.

into cover sets: the base is the first layer, the second layer consists of its neighbours, etc. The "thickness" of the branches and stems are estimated as the number of cover sets in the layers $2-6$. The relative size of the sets at the base of a branch is the quotient of the thicknesses of the branch and stem. Furthermore, the size of the sets decreases along the branch from the base to the tip to the user-defined minimum.

\subsection{QSM of individual trees}

From the tree extraction we have the point clouds of individual trees and the relative size of the cover set for every point, based on the likely sizes of local details. For each tree the QSM is reconstructed separately with the following steps. First generate a random cover with a varying set size. Next define the base of the stem as the bottom $20 \mathrm{~cm}$ layer of the point cloud and update the neighbour relation to make the whole tree connected. Then segment the tree into its stem and branches. Finally fit cylinders to the segments.

All steps until the cylinder fitting are similar to those described in the tree extraction phase. The cover parameters are $r_{2}$ and $d_{2}$, which give the maximum set sizes, and $f$, which gives the fraction of the minimum and maximum set sizes. The local sizes are calculated from these parameters. For the oak plot we used the values $d_{2}=6 \mathrm{~cm}, r_{2}=6.6 \mathrm{~cm}, n_{1}=3, f=0.25$, and for the eucalyptus plot we used the larger values $d_{2}=8 \mathrm{~cm}$, $r_{2}=8.8 \mathrm{~cm}, n_{1}=3$, and $f=0.333$. We always made five QSMs per tree to estimate the inherent modelling variations due to the randomness in cover generation. Furthermore, if the DBH of the first QSM was under $80 \%$ of the stem diameter used in the stem location process, then we progressively added $1 \mathrm{~cm}$ to $d_{1}$ and $r_{1}$ until the DBH of the QSM is over the threshold.

The refining of the segmentation is exactly the same as explained above, but there are two additional steps at the end. First, very small segments are removed if they do not have child segments and if their distance from the parent is not much more than the radius of the parent. Second, often some part of the real base of a segment is left to the parent segment forming quite large ledges. These ledges can make cylinder fits at their location too large. Thus we try to remove these ledges partly or entirely (see Appendix of Raumonen et al. 2013). The refining process can substantially reduce the number of segments and the maximum branching order so that the final segmentation usually corresponds well to the real branching structure.
2.6.1 Cylinder fitting In the last step of the QSM reconstruction, cylinders are fitted to the segments. Here we introduce another input parameter $l$, which controls the relative length of the cylinders, i.e., the ratio of the length and radius of the cylinder. Shorter cylinders can better approximate the local size and curvature of the branches but, especially with small branches, noise, an incomplete point cover, etc., can make them also more sensitive to errors. Longer cylinders are less likely to be affected by such factors, but they can be too large due to the curvature or the tapering of the branch. For both plots we used the value $l=3$.

The reconstruction proceeds according to the branching order; first the stem, then the first-order branches, etc. Each segment is first divided into subregions so that their approximate relative length is close to $l$. The radius is estimated as the median of the point distance from the approximated axis, which in turn is estimated as the vector from the region's base to the region's top.

Cylinders are fitted to the regions by least squares. The fitting is renewed with the farthest points from the cylinder surface removed as outliers. We accept the fitted cylinder if i) the radius is under three times the initial estimate, ii) the angle between the initially estimated axis and fitted axis is under $35^{\circ}$, and iii) the mean absolute distance to the cylinder's surface is smaller than the initial estimate for the radius.

The radii of the cylinders along the branch are corrected locally by imposing maximum and minimum taper curves. Details for this are presented in Calders et al. (2014). Finally the starting points are also improved to close possible gaps between cylinders by extending the starting point into the plane defined by the parent cylinder's end circle.

\subsection{Sensitivity analysis}

In this kind of reconstruction there usually are some parameters whose values must be given and which can affect the final results greatly. A useful and reliable method should not be sensitive to the exact values of these parameters, and a robust method should work even with relatively large changes in the values.

The important parameters in the tree extraction step are the cover parameter $d_{1}$ and $\alpha, \kappa, \delta$, and $\lambda$ used in the stem locating process (see Sec. 2.5.1). Table 1 shows the values given to these parameters. For $\kappa$ the value in the table is the numerator in Eq. 2. Furthermore, due to randomness, the covers for different runs are always different, which can have effects on the stem locating. Thus we have 6 variables and we ran the stem locating with all possible 1296 combinations for the oak plot. We counted the number of big stems found inside a $20-\mathrm{m}$ circle and compared it to the visually determined reference value. In all cases all reference stems were found inside the circle, and only one reference stem was missing in a few cases outside the circle. Thus the stem locating, at least for the oak plot, seems to be very robust and not very sensitive to relatively large changes in different parameter values.

\begin{tabular}{|c|c|c|}
\hline Parameter & Values & Units \\
\hline Cover set diameter $d_{1}$ & $8 ; 10 ; 12 ; 14$ & $\mathrm{~cm}$ \\
Number of covers & 4 & \\
"Normal angle" $\alpha$ & $60 ; 70 ; 80$ & degrees \\
"Component size" $\kappa$ & $0.1 ; 0.2 ; 0.3$ & \\
"Component distance" $\delta$ & $10 ; 20 ; 30$ & $\mathrm{~cm}$ \\
"Component length" $\lambda$ & $0.5 ; 1 ; 1.5$ & $\mathrm{~m}$ \\
\hline
\end{tabular}

Table 1 . Parameters in the sensitivity analysis of stem locating.

Other important parameters are $d_{2}$ and $l$ for reconstructing the QSMs. There are already some published results on these (Calders 
et al. 2014) and we are publishing soon a more detailed analysis on these, so we do not present a detailed analysis here. We only mention here that the resulting QSM and particularly its branch volume can be somewhat sensitive to the chosen values of $d_{2}$ and $l$. Usually the volume increases as we increase the values of these parameters, so they should be quite small. On the other hand, making them too small makes the results unstable.

\section{RESULTS}

\subsection{The oak plot}

We made five different covers for the tree extraction part $\left(d_{1}=8\right.$ $\mathrm{cm}, r_{1}=9 \mathrm{~cm}, n_{1}=3$ ) and for each of these cases we made five different QSMs per extracted tree $\left(d_{2}=6 \mathrm{~cm}, r_{2}=6.6 \mathrm{~cm}\right.$, $\left.n_{2}=3, f=0.25\right)$. From the modelled volume we computed the dry masses using the density and the reference dry mass computed from the empirical relation given in Eq. 1 using the DBH of the QSMs. Fig. 7 shows the average modelled masses from five different QSMs for each tree for one of the extraction cases and compares them to the reference values (the error bars show two standard deviation of the means from the five QSMs). The average relative absolute error for the five tree extraction cases varies from $23.7 \%$ to $25.5 \%$, and the total plot level biomass overestimation varies from $15.3 \%$ to $18.8 \%$. All the 15 trees inside the plot were extracted and modelled for each case. The total modelling time for filtering, tree extraction, and one QSM per tree was about $1 \mathrm{~h} 40 \mathrm{~min}$ for each case (MATLAB, MacBook Pro, 2.8GHz, 16GB). With five QSMs per tree, the total modelling time was about $4 \mathrm{~h}$ for each case.

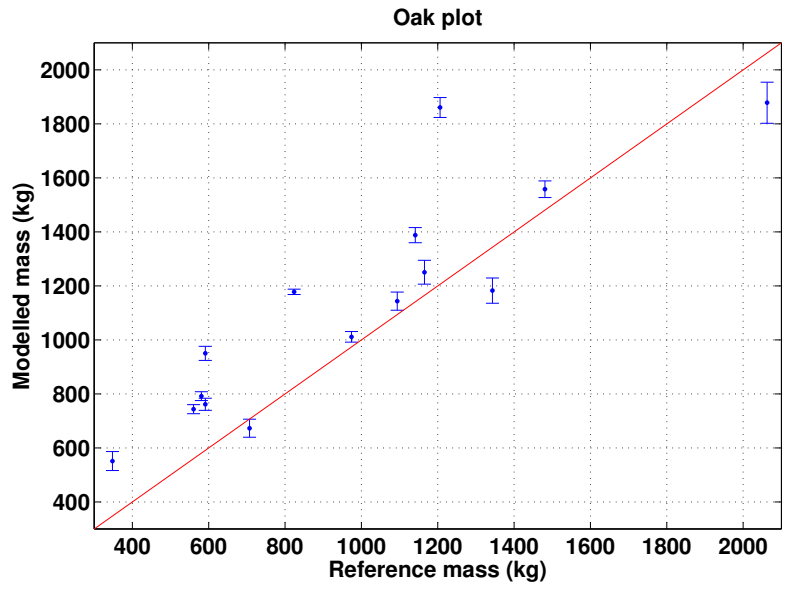

Figure 7. Comparison of modelled individual tree masses for the oak plot. The red line is the 1:1 line, the blue dots are averages and the error bars denote two times the standard deviation computed from 5 different QSMs.

\subsection{The eucalyptus plot}

We used one cover for the tree extraction part $\left(d_{1}=10 \mathrm{~cm}\right.$, $\left.r_{1}=12 \mathrm{~cm}, n_{1}=3\right)$ and made five QSMs per tree $\left(d_{2}=8 \mathrm{~cm}\right.$, $r_{2}=8.8 \mathrm{~cm}, n_{2}=3, f=0.333, l=3$ ). From the modelled volume we computed the dry masses using the density and the reference dry mass was computed from destructively measured fresh weight for 27 trees. Fig. 8 shows the average modelled masses from five different QSMs for each tree and compares them to the reference values (the error bars show two standard deviation of the means from the five QSMs). Fig. 9 shows the tree segmented point cloud and QSMs. From visual inspection we can say that every tree inside the plot was extracted and modelled.
However, in the plot there are some multi-stem trees, whose stem bifurcates very close to ground. Most of these were extracted as single trees, such that one of the stems was segmented as the stem and the others as big branches. A few reference trees were multistem trees, where the reference mass was measured for each stem. In these cases we added the reference masses together to give the reference for the one modelled tree. The average relative absolute error was about $28.5 \%$ and the error in the total biomass of the reference trees was about $8.5 \%$. The total modelling time for filtering, tree extraction, and one QSM per tree was about $2 \mathrm{~h} 40$ min (MATLAB, MacBook Pro, 2.8GHz, 16GB). With five QSMs per tree, the total modelling time was about $9 \mathrm{~h}$.

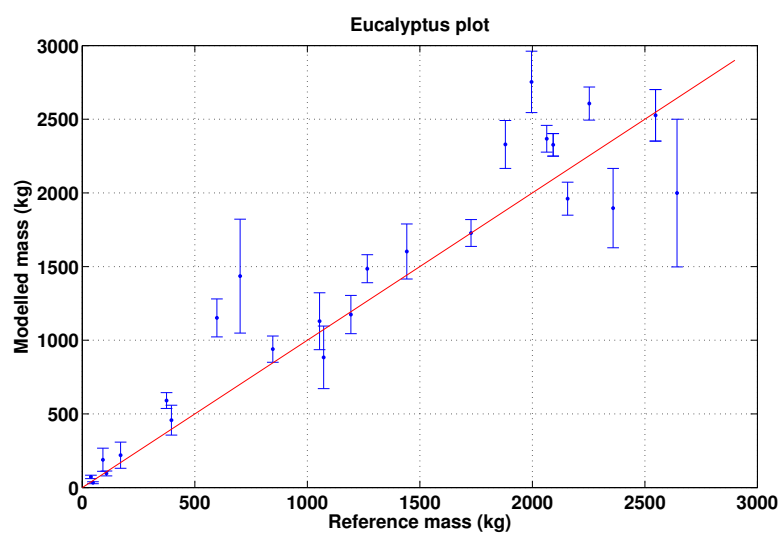

Figure 8. Comparison of modelled individual tree masses for the eucalyptus plot. The red line is the 1:1 line, the blue dots are averages and the error bars denote two times the standard deviation computed from 5 different QSMs.

\section{DISCUSSION}

The results show that the automatic tree extraction and QSM reconstructions are fast, robust, and accurate. The computation time for the filtering, tree extraction, and QSM reconstructions was altogether a few hours for both plots.

All the trees inside the oak plot were found and correctly extracted except for a few small errors with some small branches. In the case of the eucalyptus plot all the trees were also extracted, but for some multi-stem trees there were bigger errors for separating the trees correctly, which appears in Fig. 8 as bigger errors and error bars. The heuristic stem location process was shown to be robust and insensitive to the four parameters used in the process as quite large changes in the parameter values did not affect the results. We used two completely different plots and scanners, and different scanning resolutions, which further corroborates the generality and robustness of the method.

The computed tree and plot level biomass were close to the reference values. Taking into account all the uncertainties in the reference measurements, such as the wood density and dbh measurements, it is possible that the differences could be even smaller. In Calders et al. (2015) the same eucalyptus plot was modelled with the same QSM method; however, some details may have been different, the tree extraction was not automatic, and different cover sizes were used. Their modelled biomass results were similar to the ones obtained here, as expected.

These plots were not very large or dense as only five scan locations were enough to map both plots accurately, but the tests provide a proof-of-concept. The oak plot was also leaf-off making it much easier to see upper branches. With leaf-on conditions, the 


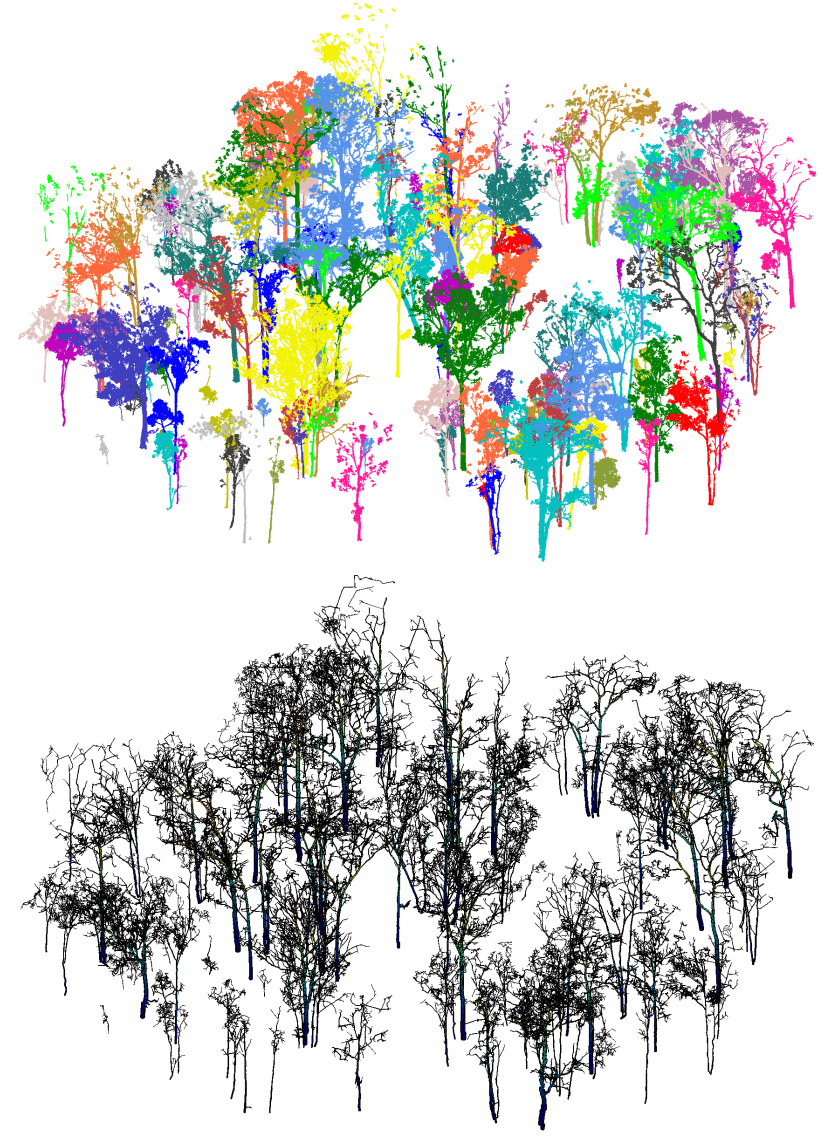

Figure 9. The extracted trees (top) and their QSMs (bottom) for the eucalyptus plot.

visibility would be poorer and we can imagine forest plots with much more understory and higher stem density. Plots that have lots of dissimilar tree species can be also challenging as different modelling parameters may be optimally suited for each species. These more challenging cases need more testing, validation, and further method development. However, these two plots can still be used for further testing and validation, and for example the multi-stems of the eucalyptus plot require modification to the algorithm to recognise them as separate trees, if needed.

In future, and to some extent already, the scanning is possible to do with mobile scanners, which means that large forest areas can be scanned more quickly and comprehensively. Our method is a good starting point for measuring the forest structure from these big data sets as the method scales up easily.

\section{CONCLUSIONS}

In this paper we have presented the first method that automatically extracts and reconstructs the volumes and 3D structures of all the individual trees in massive TLS point clouds. The method is a development of our earlier published method extracting and reconstructing a single tree from point cloud data. With two different types of plots and two different scanners, we have shown that the method is accurate, robust, and fast.

Automatic tree extraction has been a big bottleneck for the largescale use of TLS and reconstruction methods for estimating tree biomass and structure. We have demonstrated here that automatic and nondestructive biomass and structure estimation is possible in certain types of forests. Considerable further development is still needed, but this method opens up numerous possibilities in the remote sensing of forests and their applications.

\section{ACKNOWLEDGEMENTS}

This study was financially supported by the Academy of Finland project Modelling and applications of stochastic and regular surfaces in inverse problems and the Finnish Centre of Excellence in Inverse Problems Research.

\section{REFERENCES}

Åkerblom, M., Raumonen, P., Kaasalainen, M., and Casella, E. 2015. Analysis of geometric primitives in quantitative structure models of tree stems. Submitted to Remote Sensing.

Åkerblom, M. 2014. 3D forest information. www.youtube.com/watch?v=wANRdliE1zQ\&spfreload=10

Calders, K., Armston, J., Newnham, G., Herold, M., and Goodwin, N. 2014. Implications of sensor configuration and topography on vertical plant profiles derived from terrestrial lidar. Agricultural and Forest Meteorology, 194, pp. 104-117.

Calders, K., Newnham, G., Burt, A., Murphy, S., Raumonen, P., Herold, M., Culvenor, D., Avitabile, V., Disney, M., Armston, J., and Kaasalainen, M. 2015. Non-destructive estimates of aboveground biomass using terrestrial laser scanning. Methods in Ecology and Evolution, 6(2), pp. 198-208.

Dassot, M., Colin, A., Santenoise, P., Fournier, M., and Constant, T. 2012. Terrestrial laser scanning for measuring the solid wood volume, including branches, of adult standing trees in the forest environment. Computers and Electronics in Agriculture, 89, pp. 86-93.

Eysn, L., Pfeifer, N., Ressl, C., Hollaus, M., Grafl, A., and Morsdorf, F. 2013. A Practical Approach for Extracting Tree Models in Forest Environments Based on Equirectangular Projections of Terrestrial Laser Scans. Remote Sensing, 5(11), pp. 5424-5448.

Hackenberg, J., Morhart, C., Sheppard, J., Spiecker, H., and Disney, M.. 2014. Highly Accurate Tree Models Derived from Terrestrial Laser Scan Data: A Method Description. Forests, 5(5), pp. 1069-1105.

Hopkinson, C., Chasmer, L., Young-Pow, C., and Treitz, P. 2004. Assessing forest metrics with a ground-based scanning lidar. Canadian Journal of Forest Research, 34(3), pp. 573-583.

Kaasalainen, S., Krooks, A., Liski, J., Raumonen, P., Kaartinen, H., Kaasalainen, M., Puttonen, E., Anttila, K., and Mäkipää, R. 2014. Change Detection of Tree Biomass with Terrestrial Laser Scanning and Quantitative Structure Modelling. Remote Sensing, 6(5), pp. 3906-3922.

Maas, H. -G., Bienert, A., Scheller, S., and Keane, E. 2008. Automatic forest inventory parameter determination from terrestrial laser scanner data. International Journal of Remote Sensing, 29(5), pp. 1579-1593.

Raumonen, P., Kaasalainen, M., Åkerblom, M., Kaasalainen, S., Kaartinen, H., Vastaranta, M., Holopainen, M., Disney, M., and Lewis, P. 2013. Fast Automatic Precision Tree Models from Terrestrial Laser Scanner Data. Remote Sensing, 5(2), pp. 491-520. 